\title{
Evaluation of Automation to Increase Efficiency in the Dissolution Lab
}

\author{
Jonathan Kretz* and Kirby Wong-Moon \\ Analytical R\&D, Amgen Inc., Thousand Oaks, CA 91320
}

e-mail:jkretz@amgen.com

\begin{abstract}
This paper presents a comprehensive evaluation process and implementation case study results for the migration of a pharmaceutical development dissolution testing laboratory to fully automated systems to improve efficiency. Three classes of dissolution systems were evaluated: manual dissolution baths, ultraviolet (UV) online systems, and fully automated systems. The comprehensive analysis clearly shows that the fully automated system requires the lowest total analyst time to perform the dissolution experiments. Also presented are several additional factors to consider when evaluating the right type of dissolution system for a particular lab. The results of this case study show the benefit of using a fully automated dissolution system for a design of experiment (DOE) study.
\end{abstract}

\section{INTRODUCTION}

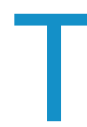

he pharmaceutical industry is in the midst of one of the greatest periods of change in its history. With health insurance reform reducing the pricing of drugs for pharmaceutical companies and the increased demands of clinical trials, the return on investment for research and development is steadily decreasing. As a whole, the industry has been driven to find new ways to reduce cost and rethink its strategy. Every department from the top down has been tasked with finding ways of being more efficient in this new era. Automation in the pharmaceutical development laboratory offers great potential for increased efficiency and reduced laboratory errors, and has been a topic of recent interest. In this paper, the writers discuss the evaluation process and preliminary implementation results for a pharmaceutical development laboratory to migrate dissolution testing toward fully automated systems to improve efficiency.

\section{EXPERIMENTAL}

First, we must define the pharmaceutical development laboratory for the purpose of this discussion. The typical pharmaceutical development laboratory contains a wide variety of instruments spanning multiple functions and platforms. The scientists in these labs perform a variety of roles rather than specializing in a single discipline, such as dissolution testing or assay testing. This is important to consider since a scientist in the laboratory may only perform dissolution testing once every month or longer and would not be expected to be as efficient as a scientist dedicated to dissolution testing. It is also important to note that the dissolution footprint is not as large as in a dedicated high-throughput dissolution laboratory. The number of dissolution baths are limited and divided between GMP and non-GMP usage.

Second, we must define the dissolution systems that we evaluated to find the best fit for our lab. The first system

${ }^{*}$ Corresponding author. was the manual dissolution bath (e.g., Distek Evolution 6300 with a syringe autosampler or Agilent VK7025 with a peristaltic autosampler). The second system can perform UV online testing (Distek 6300 with Agilent UV 8453 or Agilent VK7025 with Cary 50 UV). The third system was a fully automated Sotax AT-70 system. The writers will discuss their views on the pros and cons of each of the three systems in the evaluation.

Note that numerous other instruments that behave similarly to each of these instruments, as well as other systems (such as fiber-optic), are not discussed due to time and length restrictions. This evaluation is for our particular type of pharmaceutical development laboratory. It is very possible that a different type of lab (e.g., high-throughput) will have different objectives and considerations. So when reading this paper, please consider our recommendations in their context and evaluate whether they could be applicable to your specific laboratory environment.

Lastly, we must define the dissolution test scenario that was applied to each of the systems for a fair comparison. The test was conducted using USP Apparatus 2 (paddles) at $75 \mathrm{rpm}$. The medium was $0.05 \mathrm{~N} \mathrm{HCl}$. Four samples were tested at $n=6$. The time points were $5,15,30,45$, and 60 $\mathrm{min}$. The filter was a 10- $\mu \mathrm{m}$, full-flow filter. The sample volume was one milliliter. The samples were analyzed using either high performance liquid chromatography (HPLC)UV or UV online.

\section{Manual Dissolution Bath \\ Setup}

When setting up the manual baths, there are a number of factors to consider when determining how many baths to run. Generally, the number of baths and the number of HPLC instruments available are the biggest limiting factors. Another factor is whether the time points are too close together to manually sample. The use of autosamplers makes this limitation void for most tests since they are usually set up one per bath or one per two baths. Using the rule of thumb of two baths per HPLC, our test 
was performed over a total of three days with two baths dropped each day and then analyzed on the HPLC. The third day was for the data analysis and documentation of the second test day.

The setup time for running two baths simultaneously is broken down linearly in Table 1.

\section{Run Time, Clean Up, Analysis, and Documentation}

The total run time for this analysis was $65 \mathrm{~min}$. This is based on a 1-h run, with 5 min of extra time for the last sample pull.

Bath cleaning was performed using a vessel washer, Agilent VK 905. The total time was approximately $20 \mathrm{~min}$ per bath, or 40 min total for two baths.

Analysis was performed using HPLC. The analyst time was approximately one hour including preparation and checking system suitability. The actual HPLC run was overnight, so there was no additional down time of the analyst.

Analyst time for documentation was approximately two hours. This included a review of chromatography, data import into an electronic notebook, completion of notebook entries, and submission for review.

A summary of the analyst time is found in Table 2.

\section{UV Online System}

Setup

The limitation for this analysis is the number of baths connected to the UV instrument. In our lab, there are two baths per UV instrument. The time points are not much of a factor as most systems can be adjusted to handle increments as short as $5 \mathrm{~min}$. The software is built in with a failsafe to calculate the time needed to analyze between the two baths as well. As a result, the next bath will not start until there is no possibility for interference with measurements from the first bath.

The setup time for running two baths simultaneously is broken down linearly in Table 3.

\section{Run Time, Cleanup, and Analysis}

For this analysis, the total run time was 80 min based on the 1-h run time and use of a check standard before the run. The extra time was for the last sample pull of the second bath assuming it is started after the 5-min pull of the first bath.

Bath cleaning was performed using an Agilent vessel washer. The total time was approximately 20 min per bath, or 40 min total for two baths.

\section{Table 1. Manual Dissolution Bath Setup Tasks and Times}

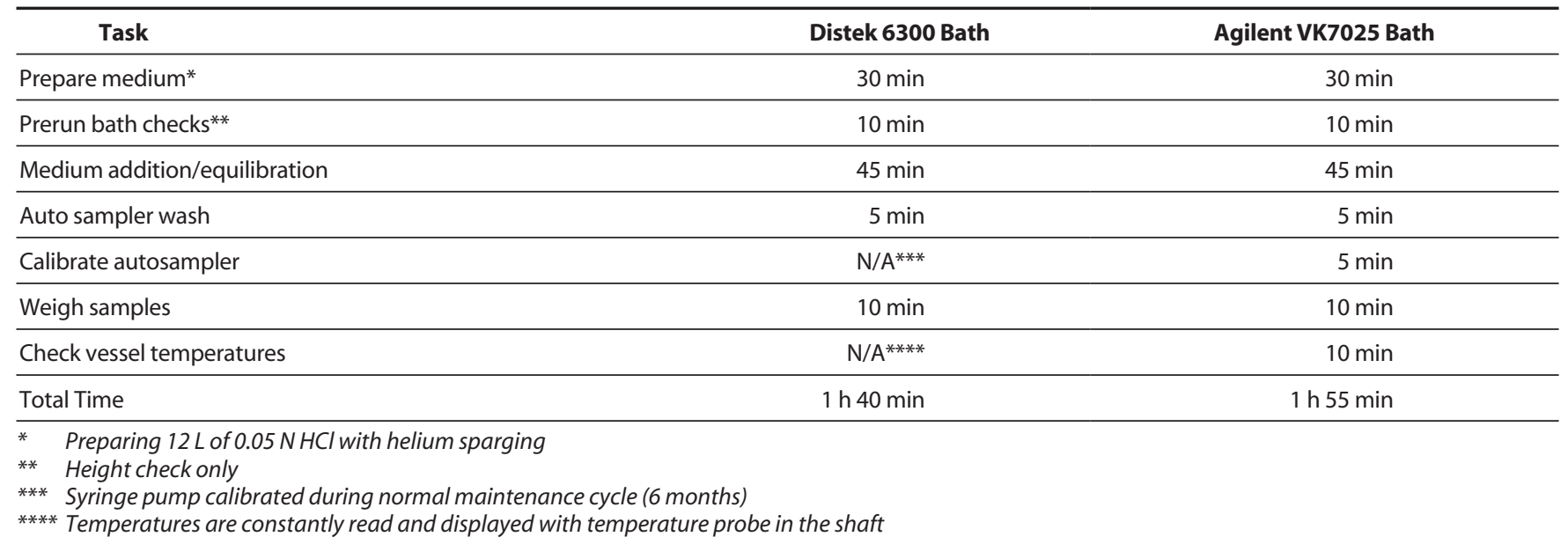

Table 2. Manual Dissolution Bath Analyst Time Summary

\begin{tabular}{|c|c|c|}
\hline Task & Distek 6300 Bath & Agilent VK7025Bath \\
\hline Setup ( 2 baths) & $1 \mathrm{~h} 40 \mathrm{~min}$ & $1 \mathrm{~h} 55 \mathrm{~min}$ \\
\hline Run time ( 2 baths) & $1 \mathrm{~h} 5 \mathrm{~min}$ & $1 \mathrm{~h} 5 \mathrm{~min}$ \\
\hline Cleanup ( 2 baths) & $40 \mathrm{~min}$ & $40 \mathrm{~min}$ \\
\hline Analyst analysis time ( 2 baths) & $1 \mathrm{~h}$ & $1 \mathrm{~h}$ \\
\hline Documentation ( 2 baths) & $2 \mathrm{~h}$ & $2 \mathrm{~h}$ \\
\hline Total Time Summary ( 2 baths) & $6 \mathrm{~h} 25 \mathrm{~min}$ & $6 \mathrm{~h} 40 \mathrm{~min}$ \\
\hline Total Time Summary (4 baths) & 12 h $50 \mathrm{~min}^{*}$ & 13 h $20 \mathrm{~min}^{*}$ \\
\hline
\end{tabular}


Analysis was performed during the run. The analyst only needed to review the data and print the results. We assign this as $15 \mathrm{~min}$.

A conservative estimate of analyst time for documentation is approximately $45 \mathrm{~min}$. This included importing data into an electronic notebook, completing the notebook write up, and submitting for review.

A summary of analyst time for the UV online system is found in Table 4.

\section{Fully Automated System Setup}

The limitation for this analysis was the number of samples that could be run by one system. One system was sufficient to complete this test, and this is what was used for the analysis. The system is equipped for UV online analysis. The time points are not a significant factor as most systems can be adjusted to handle increments as short as $5 \mathrm{~min}$.

The setup time for running four baths consecutively is broken down linearly in Table 5.

\section{Run Time, Cleanup, and Analysis}

The total run time cycle was approximately $1 \mathrm{~h} 50 \mathrm{~min}$ for this analysis. This time incorporates sparging the medium, heating the medium to temperature, running the analysis, and performing the self-cleaning aspect of the system. However, the time that an analyst is required to be present at the instrument is only about one hour since the analyst only needs to check in periodically to make sure there are no errors and review the results. This is aided by to the fact that there is a video camera that records the runs.

Cleaning the bath is incorporated in the run time.

Analysis was performed during the run. The analyst only needed to review the data and print the results. We assigned 40 min to review results for all four baths and ensure that all information was correct before printing.

Analyst time for documentation was approximately 45 min on the conservative side. This included importing data into the electronic notebook, completing the write up, and submitting for review.

A summary of analyst time for the fully automated system is found in Table 6.

\section{DISCUSSION}

There are multiple ways to break down and evaluate all of this information. One of the most important factors is

Table 3. UV Online System Setup Tasks and Times

\begin{tabular}{|c|c|c|}
\hline Task & Distek 6300 with Agilent 8453 & Agilent VK7025 with Cary 50 \\
\hline Prepare medium* & $30 \mathrm{~min}$ & $30 \mathrm{~min}$ \\
\hline Prerun bath checks ${ }^{* *}$ & $10 \mathrm{~min}$ & $10 \mathrm{~min}$ \\
\hline Medium addition/equilibration & $45 \mathrm{~min}$ & $45 \mathrm{~min}$ \\
\hline Wash sample lines & $15 \mathrm{~min}$ & $10 \mathrm{~min}$ \\
\hline Calibrate flow rate & $15 \mathrm{~min}$ & $5 \mathrm{~min}$ \\
\hline Weigh samples & $10 \mathrm{~min}$ & $10 \mathrm{~min}$ \\
\hline Check vessel temperatures & $N / A^{* * *}$ & $10 \mathrm{~min}$ \\
\hline Run blank and standards & $10 \mathrm{~min}$ & $10 \mathrm{~min}$ \\
\hline Total Times & $2 \mathrm{~h} 15 \mathrm{~min}$ & $2 \mathrm{~h} 10 \mathrm{~min}$ \\
\hline
\end{tabular}

Table 4. UV Online System Analyst Time Summary

\begin{tabular}{|c|c|c|}
\hline Task & Distek 6300 with Agilent 8453 & Agilent VK7025 with Cary 50 \\
\hline Setup ( 2 baths) & $2 \mathrm{~h} 15 \mathrm{~min}$ & $2 \mathrm{~h} 10 \min$ \\
\hline Run time ( 2 baths) & $1 \mathrm{~h} 20 \mathrm{~min}$ & $1 \mathrm{~h} 20 \mathrm{~min}$ \\
\hline Cleanup (2 baths) & $40 \mathrm{~min}$ & $40 \min$ \\
\hline Analyst analysis time ( 2 baths) & $15 \min$ & $15 \min$ \\
\hline Documentation ( 2 baths) & $45 \mathrm{~min}$ & $45 \mathrm{~min}$ \\
\hline Total Time Summary ( 2 baths) & $5 \mathrm{~h} 15 \mathrm{~min}$ & $5 \mathrm{~h} 10 \mathrm{~min}$ \\
\hline Total Time Summary (4 baths) & 10 h 30 min* & 10 h 20 min* \\
\hline
\end{tabular}


Table 5. Fully Automated System Setup Tasks and Times

\begin{tabular}{|c|c|}
\hline Task & Sotax AT70 with Agilent 8453 \\
\hline Prepare medium* & $40 \min$ \\
\hline Prerun bath checks** & N/A \\
\hline Add/equilibrate medium & $N / A^{* * *}$ \\
\hline Wash sample lines & $10 \mathrm{~min}$ \\
\hline Calibrate flow rate & N/A \\
\hline Weigh samples & $40 \mathrm{~min}$ \\
\hline Check vessel temperatures & $N / A^{* * *}$ \\
\hline Run blank and standards & $10 \mathrm{~min}$ \\
\hline Total Times & $1 \mathrm{~h} 40 \mathrm{~min}$ \\
\hline \multicolumn{2}{|c|}{$\begin{array}{l}\text { * Preparing } 24 \mathrm{~L} \text { of } 0.05 \mathrm{~N} \mathrm{HCl} \text {; note the volume can be reduced due to the } \\
\text { system's ability to dilute the medium. }\end{array}$} \\
\hline \multicolumn{2}{|c|}{ * Set at system calibration intervals } \\
\hline
\end{tabular}

the analyst time on each different system, which is summarized in Figure 1.

Based on these data alone, it would seem that automation is the best choice. However, we must consider the pros and cons for each type of system, the limitations of each system, and the type of lab into which we are fitting these systems.

\section{Manual Dissolution Bath}

Manual systems are the foundation of most dissolution laboratories. The operational knowledge is lower as the analyst does not need to remember different software operations. This is an advantage for labs where the analyst does not routinely run the dissolution test. The test methodology is universal and therefore is relatively easy to transfer from one laboratory to another.

Manual systems are the most time consuming for analysts as seen in Figure 1. There are ways to run some items in parallel, and experienced analysts are able to run four baths at the same time, which is a considerable time saver. However, for a development lab that does not specialize in dissolution, this is not a realistic expectation. Therefore, it is still necessary for an analyst to devote two days to run four baths. This time burden is a concern and highlights the need to improve efficiency in the dissolution lab.

\section{UV Online System}

UV online systems have an advantage over manual systems in that the analysis is automatically completed upon the completion of the run itself. It is time consuming, but there are enough activities that can be run in parallel to reduce the time for an analyst to one day of testing to complete the four baths. Most labs have one form or another of this type of system. This lends the ability to transfer the methodology between labs, though sometimes translations with different software programs are needed.
Table 6. Fully Automated System Analyst Time Summary

\begin{tabular}{|c|c|}
\hline Task & Sotax AT 70 with Agilent 8453 \\
\hline Setup (4 baths) & $1 \mathrm{~h} 40 \mathrm{~min}$ \\
\hline Run time (each bath) & $1 \mathrm{~h}^{*}$ \\
\hline Cleanup (each bath) & N/A \\
\hline Analyst analysis time (4 baths) & $40 \mathrm{~min}$ \\
\hline Documentation (4 baths) & $45 \mathrm{~min}$ \\
\hline Total Time Summary (4 baths) & $4 \mathrm{~h} 5 \mathrm{~min}^{* *}$ \\
\hline
\end{tabular}

The challenges of the UV online system are more significant than for the manual system, and we will focus on the three main ones of our lab. The first issue is that not all samples can utilize this system due to UV interferences from excipients and must therefore be analyzed by HPLC. The second issue is the learning curve for the software. Furthermore, if the analyst does not use the software on a regular basis, then some of that learning will be repeated. The third issue is that in the typical development lab, samples are not coming in everyday for dissolution testing. As a result, the systems will not be used often, and therefore it could take more time to bring a system back up for operation due to inactivity.

\section{Fully Automated System}

Fully automated systems are the clear winner for the amount of time an analyst needs to spend on the test. When used often, the systems run very well. Furthermore, from an instrument footprint perspective, one fully automated system can easily take the place of multiple manual baths. In addition to the UV online capability, samples can also be collected in HPLC vials. In fact, we actually run into a limitation on the number of HPLC instruments available to analyze all the samples that the fully automated system collects. A 16-h overnight run of eight sample batches ( $2 \mathrm{~h}$ per batch) would produce 240 individual samples for analysis (30 samples per batch), which would require three HPLC instruments to be prepared and ready to analyze.

Although there are clear advantages in terms of analyst time and reduced footprint, there are several disadvantages that are similar to the UV online systems. The first disadvantage is that the fully automated system is complex with a lot of capabilities. The problem is that the more features you have, the more errors that can be generated. In our lab, we ran into multiple errors where we needed to do a power cycle to clear the errors. The second disadvantage is the very steep learning curve for the software, which is further exacerbated if the analyst does not use the software on a regular basis. The third improperly cleaned lines. Although this is the same concern 


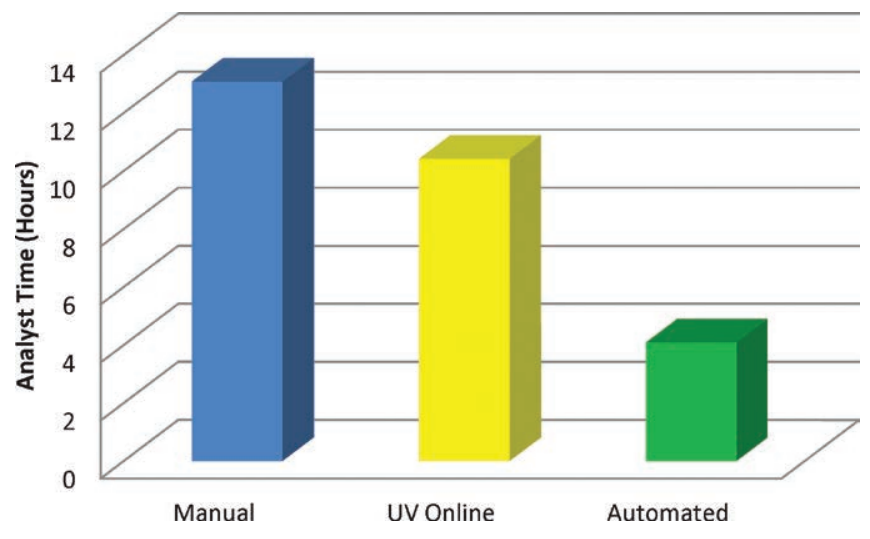

Figure 1. Total analyst time to run four baths for each type of dissolution system.

as with the UV online system, it disadvantage is that the lack of a consistent sample throughput leads to periods of inactivity, which could have the potential of drying out lines in the system or possible salt formation from is even more of an issue with the fully automated system due to its numerous components and features. The time to troubleshoot and bring back to operational status a system that has dried out lines, broken seals, or pumps that lost their prime could take up to a day. The final disadvantage is that the fully automated system is not universal. Development of a method on a fully automated system will potentially mean a transfer to a manual system before transferring to another lab that does not possess the technology.

\section{Implementation Case Study}

In our laboratory, we have found that the fully automated dissolution system works very well for certain types of high-volume experiments such as design of experiment (DOE) studies. These experiments can easily produce 20 or more samples that need dissolution testing, with the results being eagerly anticipated by formulation scientists. See Figure 2 for results of one such DOE experiment performed in our lab.

The dissolution bath used for the test was USP Apparatus 2 with a rotation speed of $75 \mathrm{rpm}$. The medium was $900 \mathrm{~mL}$ of $\mathrm{pH} 6.8$ phosphate buffer with $0.3 \%$ SDS. Sampling time points were $5,10,15,30,45$, and 60 min with an infinity time point $30 \mathrm{~min}$ after the 60 -min pull. This sample could use UV online technology at $275 \mathrm{~nm}$.

Dissolution testing for twelve DOE batches was completed using the automated system technology in a day and a half. Figure 2 shows example dissolution profiles for five of the batches used to evaluate the impact of roller force and API particle size on dissolution release. The information was communicated to the formulation scientists immediately for further decision making. In contrast

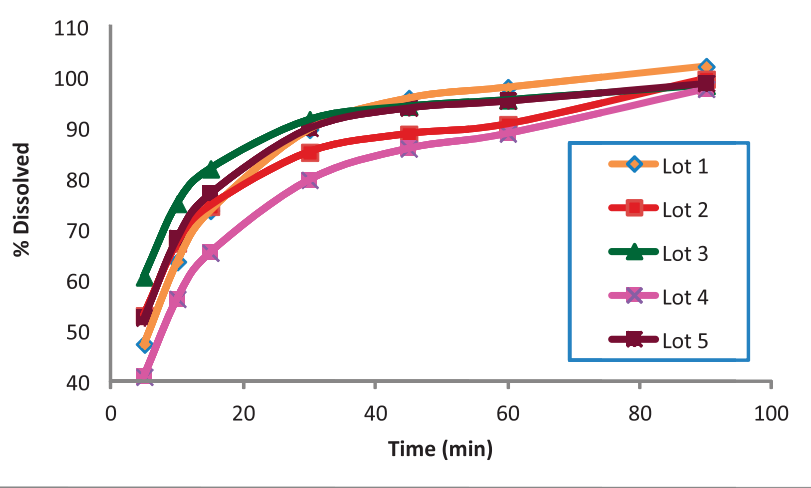

Figure 2. Results produced from DOE testing using a fully automated system.

to the fully automated testing, manual testing would have taken approximately seven days to complete. This is a clear example of improved efficiency in the laboratory.

\section{CONCLUSION}

So what type of dissolution system is the best fit for the lab? We have been evaluating this very question for quite some time now. We have seen increased usage of our fully automated system after a strong drive to retrain a core group of users in the technology. It is being run much more regularly, and the overall attitude toward the system has been very positive. We still have manual and online UV systems due to the nature of the current environment and the need to transfer methodologies.

It is difficult to believe that we will ever have a dissolution lab that no longer runs manual baths since this has been the foundation of the dissolution laboratory for some time. But with new technologies (e.g., fully automated systems directly interfaced to HPLC — see Future Work), that possibility is becoming more and more likely. For now, we must take time to assess the technology at hand and do a complete evaluation. The days of frivolously purchasing lab instruments that will not be effectively utilized are behind us. We all have to be more diligent in our expenditures and consider a greater variety of accompanying questions of transferring technologies, what will the utilization be, will the lab accept the new technology and how do we service the instruments.

\section{FUTURE WORK}

We plan to evaluate the interface of a dissolution system directly to an HPLC for analysis. This allows for a seamless flow from dissolution samples to analytical data for those samples that need to be analyzed using HPLC. Since most of the dissolution samples in our laboratory currently require offline HPLC testing, this offers significant potential for increased efficiency. 